\title{
Reaktionen auf das Offenbarungsverhalten Betroffener sexuellen Missbrauchs durch katholische Geistliche
}

Gina R. Wollinger, Lisa M. Dinkelborg, Dirk Baier

\section{Einleitung}

Vertrauen sich Kinder, die einen sexuellen Missbrauch erlebt haben, anderen Personen an, gibt ihnen das die Möglichkeit, einen andauernden Missbrauch zu beenden und weitergehende Hilfe zu bekommen. Die Reaktionen, die ein Kind daraufhin erfährt, sind bedeutend. Beispielsweise hat nach Elliott und Carnes (2001) die elterliche Unterstützung nach einem sexuellen Missbrauch einen größeren Einfluss auf die psychologische Bewältigung als Faktoren, die mit der Missbrauchstat selbst zusammenhängen. Weiter kann angenommen werden, dass es für eine/n Täter/in dann besonders einfach und die Hemmschwelle für die Tat besonders niedrig ist, wenn das Risiko, dass das Kind darüber spricht, sehr gering ist.

Im Folgenden soll das Offenbarungsverhalten der Betroffenen sexuellen Missbrauchs durch katholische Geistliche untersucht werden. Dabei wird darauf eingegangen, wann sich die Betroffenen an Dritte gewandt haben und welche Reaktionen und Folgen sie daraufhin erfahren haben. Ferner soll der Frage nachgegangen werden, worin sich die Betroffenen, die sich schon zur Tatzeit bzw. unmittelbar danach anderen anvertraut haben, von denjenigen unterscheiden, die dies erst sehr viel später oder nie getan haben. Neben den Personen aus dem sozialen Nahraum wird näher auf das Verhalten der katholischen Kirche gegenüber den Betroffenen eingegangen. Weiter werden die Häufigkeit einer Anzeigenerstattung bei der Polizei und die daraus resultierenden juristischen Folgen dargestellt.

Auf den Aufruf des Kriminologischen Forschungsinstitut Niedersachsen (KFN) an die Betroffenen zur Unterstützung bei der Aufklärung sexueller Missbrauchsfälle durch katholische Geistliche reagierten insgesamt $N=113$ Betroffene mit der Einsendung eines ausgefüllten Fragebogens. In die Auswertungen gingen schließlich die Daten von $N=104$ Personen ein, da Betroffene, welche zum Zeitpunkt der Erstviktimisierung nicht minderjährig waren, und jene, deren Täter/innen nicht eindeutig als Geistliche der katholischen Kirche klassifiziert wurden, nicht in den Analysen 
berücksichtigt werden konnten. Eine ausführliche Beschreibung von Methode, Materialien und Stichprobe dieser Studie findet sich in Kapitel 3 dieses Bandes (Fernau \& Hellmann, 2014). Nachfolgend wird die den zu berichtenden Ergebnissen zugrunde liegende Stichprobe daher nur überblicksartig beschrieben.

Die Stichprobe weist einen Altersdurchschnitt von $M=56.14$ Jahren $(S D=10.33)$ auf, wobei das Alter der Befragten zwischen 34 und 79 Jahren variierte. Häufig waren Personen der Alterskategorie 50 bis 69 Jahre in der Stichprobe vertreten. Eine Besonderheit der vorliegenden Betroffenenbefragung betrifft das Geschlechterverhältnis der Befragten. Lediglich ein Viertel der Personen, welche sich meldeten, um Angaben zu ihren sexuellen Misshandlungen durch katholische Geistliche zu machen, ist weiblich. Beim Großteil der Befragten handelt es sich damit um männliche Personen. Dieses Verhältnis findet sich auch in anderen Studien, weshalb davon auszugehen ist, dass es die tatsächlichen Geschlechterverhältnisse der Betroffenen sexuellen Missbrauchs widerspiegelt. So berichten die Deutsche Bischofskonferenz (2013) und Lueger-Schuster (2012) in ihren Forschungsberichten zu Gewalt und Missbrauch in der katholischen Kirche in Österreich, dass Jungen deutlich häufiger als Mädchen von sexualisierter Gewalt durch katholische Geistliche betroffen waren.

\section{Offenbarungsverhalten}

Im Folgenden wird das Offenbarungsverhalten der Befragten näher untersucht. Dabei soll dargestellt werden, zu welchem Zeitpunkt die Betroffenen zum ersten Mal über den Missbrauch gesprochen und an welche Personen sie sich gewandt haben.

\section{II.1 Zeitpunkt der Offenbarung}

Der sexuelle Missbrauch im Kindesalter ist eine traumatische Erfahrung, die in den meisten Fällen nicht verschwiegen wurde. In der Befragung gibt mit $92.3 \%$ fast jede bzw. jeder Betroffene an, sich bezüglich des sexuellen Missbrauchs jemandem anvertraut zu haben. Lediglich acht Befragte (7.7\%) äußern, die sexuellen Missbrauchserfahrungen bis zum Befragungszeitpunkt noch nie thematisiert zu haben.

Da der Missbrauch zum Befragungszeitpunkt im Mittel 45 Jahre zurück lag, erscheint es sinnvoll, näher zu differenzieren, wann die Betroffenen 
zum ersten Mal über das Erlebte gesprochen haben. Der Fragebogen bot die Möglichkeit anzugeben, ob man sich direkt nach dem Missbrauch, ca. ein Jahr, zwei bis fünf Jahre, fünf bis zehn Jahre oder mehr als zehn Jahre nach der Tat jemandem anvertraut hat, wobei Mehrfachantworten möglich waren. Im Folgenden wird unabhängig von der Wahl der Vertrauensperson der früheste Zeitpunkt gezählt, an dem sich eine betroffene Person öffnete.

Die Daten zeigen, dass die Hälfte der Befragten $\left(53.1 \%, N=96^{1}\right)$, die sich bis zum Befragungszeitpunkt jemandem anvertraut haben, erst mehr als zehn Jahre nach der Tat zum ersten Mal ein Gespräch über den sexuellen Missbrauch suchte. Innerhalb der Zeitspanne unter zehn Jahren findet sich, dass der zweithäufigste Moment, in welchem sich die Befragten zum ersten Mal öffneten, derjenige direkt nach dem Missbrauch darstellte $(25.0 \%)$. Wer nicht unmittelbar nach der Tat darüber sprechen konnte, dem gelang dies erst nach sehr vielen Jahren: Nur vier Befragte (4.2\%) haben innerhalb eines Jahres nach der Tat das erste Mal über die Erlebnisse gesprochen, weitere sieben $(7.3 \%)$ in der Zeitspanne von zwei bis fünf Jahren und zehn Befragte (10.4\%) innerhalb von fünf bis zehn Jahren nach der Tat.

Um Unterschiede in Bezug auf das Offenbarungsverhalten zu analysieren, wird im Folgenden zwischen denjenigen, die innerhalb eines Jahres (26.9\%) und den Befragten, die später das erste Mal oder nie (73.1\%) über das Erlebte sprachen, differenziert. Eine Unterteilung in die fünf bzw. sechs erhobenen Kategorien erscheint aufgrund der geringen Fallzahl, die manche Kategorien aufweisen, als nicht praktikabel.

Grund dafür, das Offenbarungsverhalten näher zu betrachten, ist die Annahme, dass sich ein frühzeitiges Anvertrauen positiv auf den weiteren Viktimisierungsprozess auswirkt, indem ein andauernder Missbrauch beendet und psychologische Hilfe schneller erfolgen kann. Die Untersuchung des KFN bietet die Möglichkeit, dies in Hinblick auf das heutige Vorliegen einer posttraumatischen Belastungsstörung und andere physische und psychische Beeinträchtigungen, die mittels der BSI-Skalen erhoben wurden (siehe Hellmann, Dinkelborg \& Fernau, 2014; Kap. 6 in diesem Band), zu prüfen. Allerdings zeigen sich keine signifikanten Unter-

1 Im Folgenden wird - wenn die Anzahl an Befragten, die zu einer Frage Angaben gemacht haben, weniger als 104 beträgt - diese Anzahl berichtet $(, N=$ "). Dadurch wird kenntlich gemacht, dass es nicht selten fehlende Werte gibt. Die Prozentangaben beziehen sich in solch einem Fall immer auf die Befragten, die eine gültige Angabe gemacht haben. 
schiede zwischen Betroffenen, die innerhalb eines Jahres nach der Tat mit jemandem zum ersten Mal gesprochen haben, und denen, die dies erst später bzw. nie taten. Jedoch sollte beachtet werden, dass das SichAnvertrauen nicht notwendigerweise das Erhalten von Hilfe und Unterstützung bedeutet. Weiter sind die angesprochenen psychologischen Krankheitsbilder komplex und somit von vielen weiteren Faktoren abhängig. Es scheint dennoch plausibel, dass das Sprechen mit Vertrauenspersonen gerade für ein Kind eine bedeutende Möglichkeit zur Beendigung und Verarbeitung des Missbrauchs darstellt, weshalb die Gründe und Umstände, die zu einem solchen Verhalten führen, näher untersucht werden sollten. Positive Auswirkungen des Sich-Öffnens ließen sich möglicherweise in Bezug auf andere Persönlichkeitsbereiche feststellen, die jedoch in der Befragung nicht erhoben worden sind (z. B. Lebenszufriedenheit).

Der aufgezeigte zeitliche Verlauf im Offenbarungsverhalten findet sich auch in Bezug zur Kontaktaufnahme der Betroffenen gegenüber der katholischen Kirche wieder. Mehr als die Hälfte der Befragten (54.4\%, $N=103$ ) hat sich selbst bezüglich des sexuellen Missbrauchs an die Kirche gewandt. Weitere 14 Personen (jeweils $6.8 \%$ ) geben an, dass Familienangehörige oder Freund/innen bzw. Bekannte die katholische Kirche über die Geschehnisse unterrichtet haben. In $32.0 \%$ der Fälle wurde der sexuelle Missbrauch nicht der Kirche berichtet. Die Meldung der Vorfälle bei der katholischen Kirche erfolgte sowohl durch die Betroffenen selbst als auch durch andere Vertrauenspersonen zumeist erst nach dem Missbrauch. Nur sechs Befragte $(8.4 \%, N=71)$ geben an, dass die Kirche während der Zeit des sexuellen Missbrauchs bzw. unmittelbar nach der ersten Missbrauchstat davon unterrichtet wurde. In den Jahren von 1949 bis einschließlich 1979 wandten sich elf Befragte oder deren Angehörige an die Kirche. Von 1980 bis 2009 vertrauten sich 18 Befragte der Kirche an und berichteten über den sexuellen Missbrauch. Einen deutlichen Anstieg der Mitteilungen an die katholische Kirche kann erst seit Beginn des großen öffentlichen Interesses im Jahr 2010 verzeichnet werden. Seitdem haben sich 41 der von uns befragten Personen oder deren Angehörige an die Kirche gewandt.

Während ein Großteil der Befragten sich im Laufe ihres Lebens Familienangehörigen und Freund/innen anvertraut hat und auch die Kirche in vielen Fällen von den Taten unterrichtet wurde, wandte sich nur knapp ein Viertel der Betroffenen persönlich an die Polizei $(22.2 \%, N=99)$. Bei 13 anderen Befragten (13.1\%) meldeten Familienangehörige oder sonstige Personen die Tat. Hervorzuheben ist, dass nur in einem Fall zeitnah zur Viktimisierung Strafanzeige erstattet wurde. Alle anderen Anzeigen gin- 
gen erst deutlich später bei Polizei und Staatsanwaltschaft ein. Bis zum Jahr 2009 wurden nur 13 Taten der Polizei gemeldet. Ab 2010 stieg die Anzeigequote: 15 Fälle wurden im Jahr 2010 der Polizei angezeigt und sechs weitere bis 2013.

Während $17.4 \%$ der betroffenen Frauen den sexuellen Missbrauch bei der Polizei anzeigten, meldeten die männlichen Befragten mit $41.3 \%$ die Tat signifikant häufiger, $\chi^{2}(1, N=98)=4.40, p=.036, \phi=.212$.

\section{II.2 Vertrauenspersonen}

Diejenigen Betroffenen, die über die Missbrauchserfahrungen bis zum Befragungszeitpunkt sprachen, konnten in der Befragung spezifizieren, wem sie sich anvertrauten (siehe Tabelle 1). Hierbei wurden vor allem Freund/innen und Bekannte $(61.5 \%, N=104)$ sowie die Partnerin bzw. der Partner (61.2\%) als Vertrauenspersonen benannt. Auch der Austausch mit anderen Betroffenen (59.3 \%) wurde häufig gewählt. Die Mehrheit der Betroffenen sexuellen Missbrauchs durch katholische Geistliche erhielt im Laufe des Lebens im Rahmen einer Psychotherapie Unterstützung bei der Verarbeitung der Erlebnisse. Im Rahmen dieser thematisierten drei von fünf Befragten die Missbrauchserfahrungen. Weitere $37.9 \%$ der Befragten nutzten das Gespräch mit einer Ärztin bzw. einem Arzt, um sich zu öffnen.

Betrachtet man, welche Personen innerhalb der Familie ins Vertrauen gezogen wurden, sind relativ starke Unterschiede zwischen den Familienangehörigen ersichtlich. Während sowohl die Mutter als auch die unter „andere Familienangehörige“ benannten Geschwister mit $49.0 \%$ bzw. $43.7 \%$ häufig als Vertrauenspersonen fungierten, wurde das Erlebte seltener $(35.5 \%)$ an den Vater herangetragen. Auch andere primäre Erziehungspersonen wurden eher selten (19.5\%) ins Vertrauen gezogen.

Öffentliche Instanzen wie Beratungsstellen (37.3\%) und Selbsthilfegruppen (13.7\%), aber auch die Polizei bzw. Staatsanwaltschaft (32.7\%) und sonstige Rechtsbeistände (27.4\%) wurden von einigen Betroffenen aufgesucht. $36.2 \%$ der Befragten wandten sich an die Öffentlichkeit, indem sie mit Journalisten/innen über die Taten sprachen.

Die/den Täter/in (7.5\%), Internetforen (27.4\%) und sonstige Personen (17.5\%), wobei beispielsweise evangelische Geistliche oder Arbeitskolleg/innen benannt wurden, wählten die Betroffenen im Vergleich eher selten für ein Gespräch über das Erlebte. 
Tabelle 1. Auswahl der Vertrauenspersonen nach zeitlichem Abstand zur Tat (Angaben in \%)

\begin{tabular}{lrrrr}
\hline & Direkt & 1-10 Jahre & 10 Jahre & Gesamt \\
\hline Freunde/innen & 6.3 & 10.4 & 44.8 & 61.5 \\
Partner/in & 1.1 & 10.6 & 49.5 & 61.2 \\
Therapeut/in & 1.1 & 5.3 & 54.7 & 61.1 \\
Andere Betroffene & 4.2 & 4.1 & 51.0 & 59.3 \\
Missbrauchsbeauftragte/r der Kirche & - & - & 55.2 & 55.2 \\
Andere Personen der Kirche & 2.1 & 5.4 & 45.3 & 52.8 \\
Mutter & 11.5 & 13.0 & 24.5 & 49.0 \\
Andere Familienangehörige & 4.2 & 2.0 & 37.5 & 43.7 \\
Arzt / Ärztin & - & 3.2 & 34.7 & 37.9 \\
Beratungsstelle & 3.2 & 1.1 & 33.0 & 37.3 \\
Journalisten/innen / Reporter/innen & - & - & 36.2 & 36.2 \\
Vater & 7.3 & 8.4 & 19.8 & 35.5 \\
Polizei / Staatsanwaltschaft & 2.1 & 3.2 & 27.4 & 32.7 \\
Personen im Internetforum & - & 1.1 & 26.3 & 27.4 \\
Rechtsbeistand & - & - & 24.5 & 27.4 \\
Andere Erziehungspersonen & 5.4 & 3.3 & 10.8 & 19.5 \\
Sonstige & 3.3 & 2.2 & 12.0 & 17.5 \\
Selbsthilfegruppe & - & 1.1 & 12.6 & 13.7 \\
\hline & & & & \\
\hline
\end{tabular}

Die Befragten, die sich an die Kirche wandten, nutzten als Ansprechpartner/in vor allem die/den zuständige/n Missbrauchsbeauftragte/n der Kirche $(62 \%, N=71)$. Zudem wurden die Erfahrungen in $39.4 \%$ der der Kirche bekanntgegebenen Fälle an den Erz- bzw. Weihbischof oder Bischofsvikar angetragen. Die drittgrößte Gruppe der angesprochenen Personen der Kirche stellen Angehörige eines Ordens dar (32.4\%). Seltener richteten sich die Befragten, die mit der Kirche Kontakt aufnahmen, an einen Priester, Pfarrer oder Kaplan (18.3\%) und nur vereinzelt wurden Diakone (2.9\%), Gemeindereferent/innen (1.9\%), Sozialarbeiter/innen (1\%) oder Lehrer/innen bzw. Chorleiter/innen (1\%) kontaktiert.

Auch bei der Auswahl der Vertrauensperson zeigen sich Unterschiede je nach der zeitlichen Nähe zum Missbrauch. In Tabelle 1 wurde aufge- 
schlüsselt, welche Kontaktpersonen die Betroffenen direkt nach der sexuellen Missbrauchserfahrung ins Vertrauen zogen und welche im Laufe von einem bis zehn Jahren bzw. erst nach über zehn Jahren informiert wurden. Wie zu erwarten, wurden in zeitlicher Nähe zum Missbrauch eher nahestehende Angehörige angesprochen. Fremde Personen, wie beispielsweise Journalisten/innen, wurden erst später kontaktiert. Auch Selbsthilfegruppen und andere Beratungsstellen stellten erst mehr als zehn Jahre nach der Tat Gesprächspartner/innen dar.

\section{II.3 Einflussfaktoren auf das Offenbarungsverhalten}

Für $72.5 \%$ der Befragten $(N=102)$ war das öffentliche Bekanntwerden und die damit verbundene mediale Debatte im Jahr 2010 eine Ermutigung dazu, über den sexuellen Missbrauch zu sprechen. Im Folgenden soll untersucht werden, welche Faktoren dafür ausschlaggebend sind, zu welchem Zeitpunkt sich ein/e Betroffene/r das erste Mal jemandem anvertraut. Die Kontaktaufnahme zu Vertrauenspersonen zur Tatzeit bzw. unmittelbar danach scheint von besonderer Bedeutung zu sein, da in diesem Fall ein noch andauernder Missbrauch beendet werden und frühzeitig psychologische Unterstützung erfolgen kann. Deshalb soll in der weiteren Analyse des Offenbarungsverhaltens zwischen zwei Zeiträumen unterschieden werden: Zum einen das Sich-Anvertrauen innerhalb eines Jahres nach dem sexuellen Missbrauch und zum anderen das Sich-Öffnen zu späteren Zeitpunkten bzw. gar nicht. Es soll dabei untersucht werden, inwiefern sozialisationsbedingte oder religiöse Faktoren bzw. die näheren Umstände der Missbrauchstat die Offenbarungsbereitschaft der Minderjährigen beeinflussten.

\section{Sozialisation der Betroffenen}

Zur Sozialisation der Betroffenen wurde das Erziehungsverhalten der Eltern bzw. der primären Erziehungspersonen erfragt. Die Befragten konnten mittels einer fünfstufigen Skala (1-nie bis 5-sehr häufig) angeben, ob verschiedene Verhaltensweisen von Eltern oder anderen Erziehungspersonen auf ihre eigene Kindheit zutrafen. Hieraus wurden drei Erziehungsstile generiert: zugewandte Erziehung, Erziehung mit leichter Gewalt und solche mit schwerer Gewalt. 
Eine deutschlandweite repräsentative Bevölkerungsumfrage des KFN von 1992 (sogenannter KFN-Viktimsurvey; siehe z. B. Wetzels, 1997), ermöglicht einen Vergleich der Erziehungsstile zu anderen Personen derselben Altersgruppe. Bei dem KFN-Viktimsurvey von 1992 wurden $N=15771$ Personen deutscher Staatsangehörigkeit befragt. Der Schwerpunkt der Studie lag auf Kriminalitäts-Betroffenenerfahrungen. Daneben wurden jedoch unter anderem auch Fragen zur eigenen Sozialisation gestellt. Diese Fragen wurden allerdings nur $n=3289$ Personen zur Beantwortung vorgelegt.

Der zugewandte Erziehungsstil wurde in der KFN-Befragung zum sexuellen Missbrauch Minderjähriger durch katholische Geistliche durch eine Mittelwertskala mit folgenden fünf Items gebildet ${ }^{2}$ : Meine Eltern / Erziehungspersonen haben...

- mich gelobt, wenn ich etwas besonders gut gemacht hatte

- mich in den Arm genommen und mit mir geschmust

- mir ruhig erklärt, wenn ich etwas falsch gemacht hatte

- mich getröstet, wenn ich traurig war

- mir etwas geschenkt bzw. mir etwas Besonderes erlaubt, wenn ich etwas besonders gut gemacht habe.

Die Mittelwerte von $M=1$ bis $M=2.34$ zeigen geringe, die zwischen $M=2.34$ und $M=3.67$ mittlere und die über $M=3.67$ hohe Zuwendung an. Der Mittelwert der Befragten insgesamt liegt bei $M=2.33$ $(S D=0.98) .12 .6 \%(N=103)$ berichten, einen hohen Grad an Zuwendung in ihrer Kindheit erlebt zu haben, $33.0 \%$ geben hierbei eine mittlere Stärke an; eine geringe Zuwendung berichten $54.4 \%$. Signifikant niedriger ist der Mittelwert mit $M=1.79(S D=0.79)$ bei Betroffenen, die ihre Kindheit in einem Heim verbracht haben, $t(99)=5.48, p<.001, d=1.11$; bei Befragten, die nicht in einem Heim lebten, liegt dieser bei $M=2.75$ $(S D=0.96)$.

Der zugewandte Erziehungsstil wurde im KFN-Viktimsurvey von 1992 in vergleichbarer Weise abgefragt, allerdings ohne das Item ,....mich getröstet wenn ich traurig war" “. ${ }^{3}$ Wird dieses bei der Skalenbildung nicht be-

2 Die Reliabilität der Skala ist mit Cronbach's $\alpha=.92$ als sehr gut einzustufen.

3 Zudem wurde in der Befragung 1992 das Item ,mir etwas geschenkt bzw. mir etwas Besonderes erlaubt, wenn ich etwas besonders gut gemacht habe" mittels zweier Items abgefragt (,mir erlaubt, länger aufzubleiben, wenn ich etwas besonders gut gemacht habe“ und „mir etwas geschenkt, wenn ich etwas besonders gut gemacht habe"). In 
rücksichtigt, beträgt der Mittelwert der Zuwendung $M=2.30$ (niedrig: $61.2 \%$, mittel: $26.2 \%$, hoch: $12.6 \%$ ). Deutlich höhere Werte ergeben sich in der Befragung von 1992: Der Mittelwert für die gesamte Stichprobe liegt bei $M=3.4$. Von einer hohen Zuwendung berichten $39.8 \%$ der Befragten, von einer niedrigen Zuwendung nur $9.2 \%$ (mittel: $50.9 \%$ ). ${ }^{4}$

Eine Maximalwertskala ${ }^{5}$ zur ,leichten Gewalt“" wurde aus folgenden drei Items gebildet: Meine Eltern / Erziehungspersonen haben...

- mit einem Gegenstand nach mir geworfen

- mich hart angepackt oder gestoßen

- mir eine runtergehauen

Sofern mindestens eines dieser Items selten zutraf, liegt das Erleben leichter Gewalt vor. Dies ist bei $87.4 \%(N=103)$ der Befragten der Fall. Die Betroffenen, die in einem Heim gelebt haben, scheinen stärker von leichter Gewalt betroffen zu sein. $93.3 \%$ derjenigen, die in einer solchen Einrichtung gelebt haben, berichten Formen leichter Gewalt, wohingegen dies $82.1 \%(N=101)$ derjenigen angeben, die nicht in einem Heim waren. Wird der Viktimsurvey des Jahres 1992 zum Vergleich herangezogen, ergibt sich wiederum ein Unterschied derart, dass Personen der Befragung zum sexuellen Missbrauch häufiger negative Erziehungserfahrungen machen mussten: So gaben nur $74.2 \%$ der Befragten des Viktimsurveys an, leichte Gewalt durch die Eltern in Kindheit und Jugend erfahren zu haben.

Der dritte analysierte Erziehungsstil „Schwere Gewalt“ wurde ebenso mittels einer Maximalwertskala gebildet. Folgende Items wurden dazu herangezogen: Meine Eltern / Erziehungspersonen haben...

der Auswertung wurde dies folgendermaßen berücksichtig: Zuerst wurde aus beiden Items der Maximalwert gebildet. Dann wurde über die vier Items der Mittelwert gebildet. Der Wortlaut der anderen drei Items sowie die Antwortvorgaben waren in der Befragung von 1992 identisch.

4 Die Altersverteilung beider Befragungen stimmt weitestgehend überein, weshalb bezüglich des Vergleichs keine Einschränkungen der Altersgruppen vorgenommen wurden. Im Viktimsurvey befinden sich Personen, die zwischen 1932 und 1976 geboren wurden (Median: 1957 geboren), in der Befragung zum sexuellen Missbrauch Personen, die zwischen 1934 und 1979 geboren wurden (Median: 1956 geboren).

5 Maximalwertskala bedeutet, dass der höchste Wert eines Items in der zugehörigen Skala berücksichtigt wird. Wenn also beispielsweise ein/e Befragte/r angibt, häufig hart angepackt oder gestoßen worden zu sein, die anderen Übergriffsformen jedoch nicht erlebt hat, geht die Antwort zum Anpacken / Stoßen in die Skala ein. 
- mich mit der Faust geschlagen, getreten oder mich gebissen

- mich mit einem Gegenstand geschlagen oder zu schlagen versucht

- mich geprügelt, zusammengeschlagen

Von diesem Erziehungsverhalten waren $65.0 \%$ der Befragten der sexuellen Missbrauchsstudie betroffen. Auch hier zeigen sich deutliche Unterschiede in Bezug auf eine Heimerfahrung. $80.0 \%(N=101)$ der Befragten, die in einem Heim ihre Kindheit verbrachten, geben schwere Gewalterfahrungen an, wohingegen $51.8 \%$ derjenigen Personen, die nicht in einem Heim lebten, dies nennen. Dieser Unterschied ist signifikant, $\chi^{2}(1$, $N=101)=8.66, p=.003, \phi=.293$. Auch unabhängig von einer Heimerfahrung sind diese Werte deutlich höher als bei Personen, die nicht von einem sexuellen Missbrauch durch katholische Geistliche betroffen sind. Im Viktimsurvey von 1992 gaben $16.2 \%$ der Befragten an, Formen schwerer Gewalt von ihren Erziehungspersonen erlebt zu haben.

Es kann somit festgestellt werden, dass das Erziehungsverhalten, welches Betroffene sexuellen Missbrauchs durch katholische Geistliche in ihrer Kindheit erfuhren, sehr viel stärker gewaltgeprägt und weniger durch liebevolle Zuwendung gekennzeichnet ist als bei anderen Personen des gleichen Alters.

Im Folgenden soll den möglichen Auswirkungen der Sozialisationserfahrungen auf das Offenbarungsverhalten nachgegangen werden. Über die Hälfte der Betroffenen (53.8 \%), die einen hohen Grad an Zuwendung von ihren Erziehungspersonen erfuhren, redeten innerhalb eines Jahres nach der Missbrauchstat mit jemandem darüber, während dies nur $29.4 \%$ der Betroffenen taten, die eine mittlere Stärke in der Zuwendung verzeichneten und nur $19.6 \%$ derjenigen, die geringe Zuwendung erfuhren. Diese Unterschiede sind signifikant, $\chi^{2}(2, N=103)=6.36, p=.042, \phi=.149$. Unterschiede zeigen sich auch in Hinblick auf die Kontaktaufnahme mit der katholischen Kirche. Diejenigen Befragten, die sich selbst oder deren Angehörige sich an die Kirche wandten, unterscheiden sich mit einem Mittelwert der Zuwendung von $M=2.47(S D=0.12)$ signifikant von solchen, die nicht eine Person der Kirche ansprachen $(M=2.04, S D=0.95)$, $t(100)=2.06, p=.042, d=0.44$. Mittelwertsunterschiede zeigen sich jedoch nicht in Bezug auf das Anzeigeverhalten bei der Polizei.

Zur Analyse des Offenbarungsverhaltens in Zusammenhang mit dem Erleben einer gewaltvollen Erziehung sollen Personen, die gar keine Gewalt erfahren haben $(10.7 \%, N=103)$, solche die nur leichte Gewaltformen erlebten $(24.3 \%)$ und Befragte, die (auch) schwere Gewalt $(65.0 \%)$ berichteten, unterschieden werden. $36.4 \%$ der Betroffenen, die keinerlei 
Gewalt in ihrer Kindheit von ihren Erziehungspersonen erlebten, sprachen innerhalb eines Jahres nach der Tat über den Missbrauch, während dies nur $24.0 \%$ der Personen mit leichten Gewalterfahrungen und $26.9 \%$ derjenigen mit schwerem Gewaltvorkommen taten. Gewalterfahrungen in der Erziehung scheinen jedoch weniger in Zusammenhang mit einer Mitteilung an die Kirche zu stehen. $81.8 \%$ derjenigen, die keine Gewalt erlebt haben, wandten sich im Laufe ihres Lebens an die Kirche. Dies taten ebenso $80.0 \%$ der Betroffenen, die leichte Gewalt von ihren Erziehungspersonen erfuhren und $62.1 \%$ der Befragten, die schwere Gewalt erlebten. In Bezug auf eine polizeiliche Anzeige lässt sich keine eindeutige Tendenz erkennen. $36.4 \%$ der Betroffenen ohne Gewalterfahrungen durch die Erziehungspersonen zeigten die Tat an, wohingegen $40.9 \%$ derjenigen mit leichten Gewaltvorkommnissen und $33.8 \%$ der Befragten, die schwere Gewalt erlebten, anzeigten.

Diese Ergebnisse deuten daraufhin, dass es einer expliziten positiven Zuwendung der Eltern bedarf, die nicht allein durch das Fehlen von Gewalt besteht, um Kinder zu ermutigen, sich mit ihren Sorgen jemandem anzuvertrauen.

Des Weiteren ist die besondere Situation des Aufwachsens in einem Heim für die Bereitschaft, die eigenen sexuellen Missbrauchserfahrungen zu berichten, zu untersuchen. Es wurden jedoch keine signifikanten Unterschiede in Bezug auf die Offenbarungsbereitschaft zwischen Minderjährigen mit und ohne Heimerfahrung ersichtlich. Ferner wurde erhoben, inwiefern die Befragten die Atmosphäre und den Umgang miteinander im Heim als respektvoll oder eher gewaltvoll beurteilen. Jedoch können auch hier keine signifikanten Unterschiede in Bezug auf das Offenbarungsverhalten gezeigt werden.

\section{Religiosität}

Da die Täter/innen katholische Geistliche waren, stellt sich die Frage nach der Rolle der Religiosität im Zusammenhang des Offenbarungsverhaltens. Benkert und Doyle (2009) weisen in Bezug auf sexuellen Missbrauch in kirchlichen Institutionen auf die Bedeutung des religious duress hin, einer besonderen Art von Angst und Zwang, welche auf der Tatsache basiert, dass religiöser Glaube an elementare Ängste, Wünsche und Hoffnungen des Menschen anknüpft. Auch betonen sie die besondere Stellung des Priesters (v. a. innerhalb der katholischen Kirche) und die Beziehung zu 
ihm, die schon an sich mit etwas Geheimnisvollem belegt sei (Benkert \& Doyle, 2009).

Um den Einfluss der Religiosität sowie der gesellschaftlichen Sonderstellung Geistlicher zu untersuchen, ist die KFN-Befragung auf unterschiedliche Faktoren religiöser Sozialisation und Teilhabe eingegangen. Dabei wird davon ausgegangen, dass der sexuelle Missbrauch zwar in einem religiösen Kontext stattfand, die Stärke der Religiosität der Betroffenen dennoch unterschiedlich ausgeprägt sein kann. Um dies zu erfassen, wird eine Mittelwertskala aus folgenden Indikatoren gebildet (siehe auch Tabelle 2; vgl. Wetzels \& Brettfeld, 2003): Wichtigkeit von Religion und Glaube in der Zeit bis zum sexuellen Missbrauch, Häufigkeit von Kirchenbesuchen, Gebetshäufigkeit und Bedeutung von Religion und Glaube in der Erziehung durch die Mutter und den Vater. Zunächst wurden die Antwortkategorien der Items so umkodiert, dass jede Indikatorvariable nur noch vier Ausprägungen hatte, wobei die eins für „nicht religiös“ und die vier für „sehr religiös“ steht (siehe Tabelle 2). Um die Stärke der religiösen Erziehung durch die Eltern nicht zu stark in die Religiositätsskala einzubeziehen, wurde in einem weiteren Schritt eine Maximalwertskala aus der Bedeutung von Religion und Glaube in der Erziehung der Mutter und des Vaters gebildet, sodass eine Variable zum Erziehungsverhalten der Eltern vorliegt. Über diese vier Variablen wurde dann eine Mittelwertskala zur Erfassung der Religiosität der Befragten gebildet. ${ }^{6}$

Die Befragten weisen insgesamt einen Mittelwert von $M=3.32$ $(S D=0.84)$ auf. Die Personen, die sich innerhalb eines Jahres jemandem anvertraut haben, sind mit einem Mittelwert von $M=3.20(S D=0.88)$ auf der Religiositätsskala etwas weniger stark religiös als diejenigen, die später oder nie über den Missbrauch sprachen $(M=3.35, S D=1.83)$. Dieser Unterschied ist jedoch nicht signifikant. 


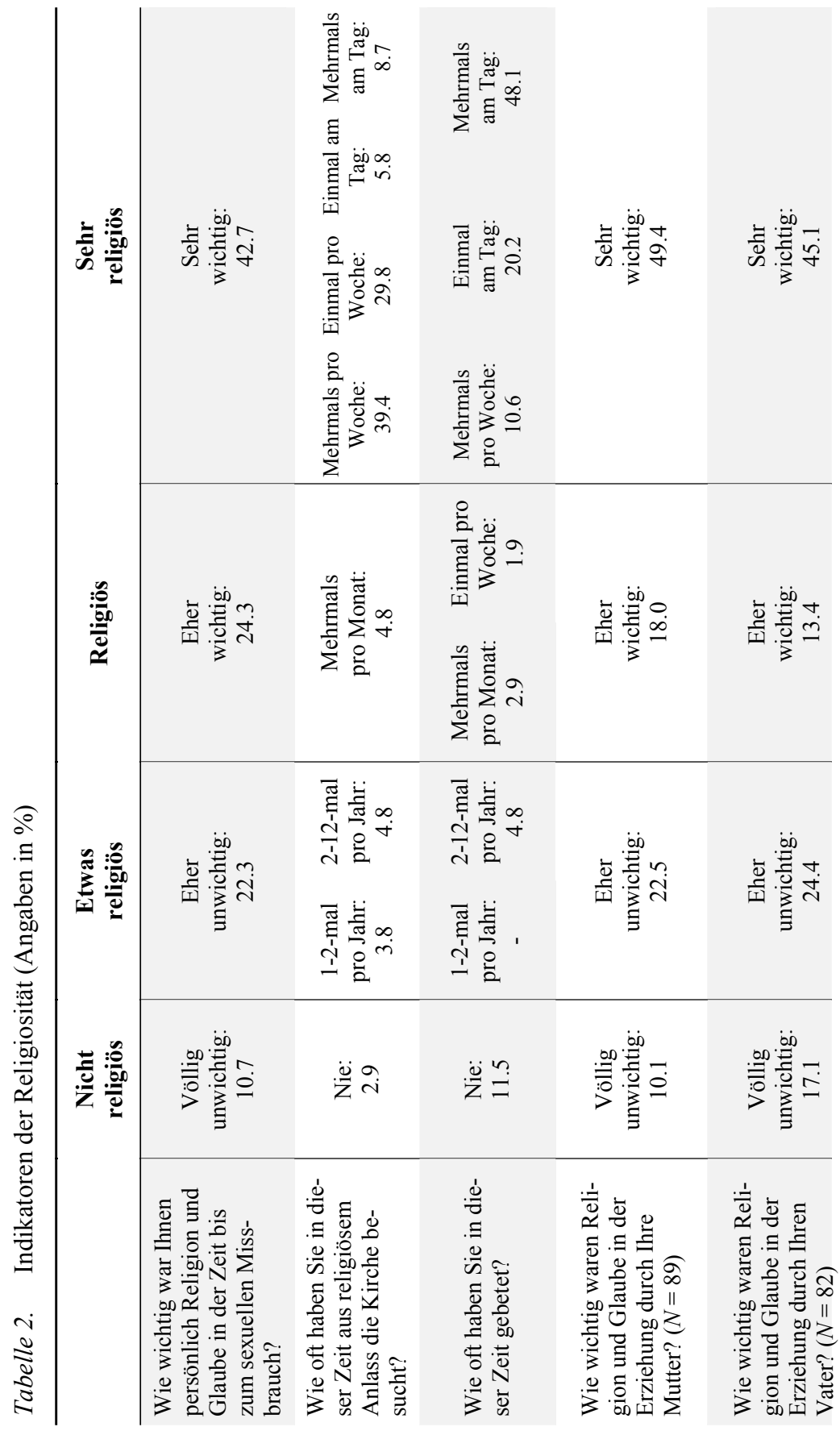


Neben der Religiosität der Betroffenen kann weiter die Religiosität der Eltern untersucht werden. Indikator dafür soll die Kirchgangshäufigkeit der Eltern sein. Diese wurde getrennt nach Mutter und Vater abgefragt. Die Variablen wurden so umkodiert, dass sie nur noch Ausprägungen von 1nicht religiös bis 4-sehr religiös annehmen. In einem weiteren Schritt wurde eine Mittelwertskala aus den beiden Variablen gebildet, die die Religiosität der Eltern angibt. ${ }^{7}$ In Bezug auf die Mutter wurde berichtet, dass sie zu $66.6 \%$ mindestens mehrmals pro Woche die Kirche besucht hat, in Bezug auf den Vater wurde dies zu $60.2 \%$ bestätigt.

Die Religiosität der Eltern von Befragten, die innerhalb eines Jahres nach der Tat über das Erlebte sprachen, war mit einem Mittelwert von $M=2.98(S D=1.25)$ etwas geringer als die derjenigen Betroffenen, die sich später oder nie jemanden anvertrauten $(M=3.17, S D=1.11)$; dieser Unterschied ist allerdings nicht signifikant.

Neben der eigenen Religiosität und der der Eltern zum Zeitpunkt des sexuellen Missbrauchs wurde die religiöse Prägung des Umfelds erhoben. Die Befragten wurden aufgefordert, den Anteil an katholischen Einwohner/innen in ihrem damaligen Wohnort zu schätzen. Dabei konnten sie angeben, ob fast alle, die Mehrheit, ungefähr die Hälfte bzw. die Minderheit der Einwohner/innen katholisch waren oder ob es kaum katholische Einwohner/innen gab. ${ }^{8} 69.3 \%$ der Befragten $(N=101)$ geben an, dass sie zur Zeit des Missbrauchs in einem Ort gelebt haben, in dem fast alle bzw. die Mehrheit der Einwohner/innen katholisch waren. Dies wird im Folgenden als katholisch geprägter Ort bezeichnet. $38.7 \%$ derjenigen, die nicht zur Tatzeit in einem katholisch geprägten Ort lebten, öffneten sich zum ersten Mal innerhalb eines Jahres nach der Tat. Dagegen vertrauten sich nur $20.0 \%$ der Betroffenen aus einem katholisch geprägten Ort einer Person in dieser Zeitspanne an. Dieser Unterschied ist signifikant, $\chi^{2}(1, N=101)=3.93, p=.047, \phi=.197$. Der Zusammenhang zwischen dem Anteil der Katholiken/innen im Wohnort und dem Offenbarungsverhalten wurde auch in einer offenen Antwort auf die Frage, warum keine Anzeige bei der Polizei erstattet wurde, verdeutlicht: „Weil es in den

7 Die Reliabilität der Skala ist mit Cronbach's $\alpha=.84$ ebenfalls als gut einzustufen.

8 Diese Einschätzung ist rein subjektiv und kann unter Umständen von der tatsächlichen Verteilung der Religionszugehörigkeit abweichen. Relevant für die Untersuchung ist jedoch nicht die tatsächliche Verteilung sondern die subjektiv wahrgenommene Dominanz bestimmter Gruppen. Dabei wird angenommen, dass die subjektive Wahrnehmung stärkeren Einfluss auf das Handeln der Person hat als die objektive Sachlage. 
sechziger Jahren im katholischen [Stadt mit ca. 300000 Einwohner/innen] nichts gebracht hätte.“

\section{Merkmale der Missbrauchstat}

Untersucht werden soll zudem, ob sich bestimmte Merkmale der Missbrauchstat auf das Offenbarungsverhalten auswirken. Der sexuelle Missbrauch kann sehr unterschiedliche Verhaltensweisen und Handlungen beinhalten. Beispielsweise können Gespräche über Sexualität, das Zeigen pornografischer Filme, aber auch direkte körperliche Übergriffe stattgefunden haben. Es zeigt sich, dass nur $20.6 \%$ der Betroffenen, die sexualisierte Gewalthandlungen erlebt haben, bei denen Oralverkehr oder Penetration stattgefunden hat ${ }^{9}$ innerhalb des ersten Jahres nach der Tat darüber das erste Mal sprachen. Von denjenigen Befragten, die solche Formen des Missbrauchs nicht erlebt haben, wandten sich $43.8 \%$ das erste Mal innerhalb eines Jahres nach dem Missbrauch an eine andere Person. Dieser Unterschied ist signifikant, $\chi^{2}(1, N=100)=5.79, p=.016, \phi=.241$. Es soll hier keinerlei Bewertung der Schwere zwischen den Missbrauchsformen vorgenommen werden. Die Daten sind lediglich ein Indiz dafür, dass bei einigen Formen von Missbrauchshandlungen eine besonders erhöhte Hemmschwelle vorliegt, darüber zu sprechen.

Exkurs: Befragte, die bis heute mit niemanden über den Missbrauch gesprochen haben

Acht Befragte geben an, bis zum Zeitpunkt der Untersuchung mit niemandem über das Erlebte gesprochen zu haben. Es handelt sich um fünf Männer und zwei Frauen (ein/e Befragte/r nannte sein Geschlecht nicht), die zwischen 1938 und 1959 geboren wurden. Bei sechs von ihnen dauerte der sexuelle Missbrauch mindestens ein Jahr an. Drei geben an, dass sie von mehr als einem Geistlichen sexuell missbraucht wurden. Die Miss-

9 Dies wurde mit folgenden Items im Fragebogen erfasst: „Ein katholischer Geistlicher hat mich aufgefordert, sein Geschlechtsteil zu berühren oder ihn anderweitig mit der Hand oder dem Mund sexuell zu erregen; hat mein Geschlechtsteil in den Mund genommen; ist mit seinem Geschlechtsteil in meinen Mund eingedrungen; ist mit seinem Finger, seiner Zunge und / oder einem Gegenstand in mich eingedrungen; ist mit seinem Geschlechtsteil in mich eingedrungen." 
brauchshandlungen sind dabei sehr unterschiedlich und reichen von dem gemeinsamen Schauen pornografischer Videos bzw. Magazine bis hin zu Penetrationen.

Auffällig ist das Verhalten der Erziehungspersonen der acht Betroffenen. Nur eine Person hat eine hohe Zuwendung erfahren und keinerlei Gewalt, sieben hingegen haben sowohl schwere als auch leichte Gewalt in ihrer Kindheit erlebt. Zum Wohnort zur Tatzeit geben vier an, dass fast alle Einwohner/innen katholisch waren und drei, dass die Mehrheit katholisch war.

Die Gründe, die diese Befragten bewegten, bis zum Zeitpunkt der Untersuchung niemanden über die eigenen Missbrauchserfahrungen in Kenntnis zu setzen, sind vielfältig. Überwiegend sind sie der Meinung, dass eine Meldung der sexuellen Missbrauchshandlungen unnütz sei. Dies wird von sechs Befragten durch die Aussage spezifiziert, dass niemand ihren Berichten über Missbrauchshandlungen durch katholische Geistliche Glauben schenken würde. Außerdem könne sowieso niemand das Ausmaß des Missbrauchs für die Betroffenen nachvollziehen, begründen die Befragten ihre Entscheidung, die Erlebnisse für sich $\mathrm{zu}$ behalten. Jede/r Zweite/r betont, aufgrund der Zugehörigkeit der/des Täters/in zur Kirche nicht über die Erlebnisse sprechen zu wollen. Drei Befragte teilen zudem mit, dass sie ihre Familien nicht belasten wollten und es sonst niemanden gab, dem sie von den Erlebnissen berichten konnten. In Übereinstimmung damit geben fünf Geschädigte an, dass sie die Erfahrungen lediglich vergessen wollten und Angst davor hatten, dass ihre Missbrauchsgeschichte an die Öffentlichkeit drang.

Eine weitere Motivation für das langjährige Schweigen könnte auch darin begründet liegen, dass es sich bei den berichteten Taten meist nicht um einzelne Ereignisse handelte, sondern um Missbräuche, die über einen längeren Zeitraum hinweg verübt wurden (siehe Hellmann, Dinkelborg, Wollinger \& Fernau, 2014; Kap. 4 in diesem Band). Gerade in diesen Fällen, in denen sich eine bestimmte Art von Beziehung zwischen der/dem Täter/in und dem Betroffenen entwickelt, fällt es schwer, das Schweigen zu brechen. Ein Mechanismus, welcher auch als „trauma bond“ (Benkert \& Doyle 2009, S. 235) bezeichnet wird.

\section{Reaktionen auf das Offenbarungsverhalten}

Vertrauen sich Betroffene sexuellen Missbrauchs nach dem Erlebten einer Person an, nimmt die Reaktion dieser Person eine besondere Stellung im 
Verarbeitungsprozess ein. So führt beispielsweise elterliche Unterstützung nach einem sexuellen Missbrauch langfristig zu einer besseren psychischen Gesundheit (Briere \& Elliott, 1994; Elliott \& Carnes, 2001). Sie verzeichnet sogar einen größeren Einfluss auf das psychische Befinden als Faktoren, die mit dem Missbrauch selbst zusammenhängen (Elliott \& Carnes, 2001).

\section{III.1 Allgemeine Reaktionen}

Die Befragten hatten die Möglichkeit anzugeben, inwiefern verschiedene Reaktionen allgemein auf sie zutrafen, wenn sie sich jemanden mitgeteilt haben. Dabei konnten sie zwischen trifft nicht zu, trifft teilweise zu und trifft völlig zu differenzieren. Die Zustimmungen in Form von teilweise und völlig sind hier in der Auswertung zusammengefasst. In einem freien Antwortformat konnten auch nicht aufgeführte Reaktionen genannt werden. Erhoben wurden damit sämtliche Antworten und Verhaltensweisen, die die Betroffenen im Laufe ihres Lebens erfahren haben. Die Reaktionen wurden jedoch nicht zugeordnet $\mathrm{zu}$ den jeweiligen Vertrauenspersonen abgefragt. Da einige Betroffene mit mehreren Personen über ihre Erfahrungen sprachen, ist es möglich, dass ein/e Befragte/r sowohl positive als auch negative Reaktionen berichtet.

Im Allgemeinen berichten die Betroffenen positiv unterstützende Reaktionen der Kontaktpersonen. In fast allen Fällen wurde den Betroffenen zugehört $(92.3 \%, N=91)$ und geglaubt $(91.6 \%, N=95)$. Drei Viertel der Befragten $(N=85)$ schilderten ebenso, dass sie emotional unterstützt worden seien und $69.7 \%(N=89)$, dass sie bei der Verarbeitung des Traumas Hilfe bekamen. $37.2 \%(N=86)$ der Befragten erhielten eine finanzielle Unterstützung.

Wenngleich in geringerer Häufigkeit als die unterstützenden Reaktionen sind die ablehnenden Verhaltensweisen als besonders drastische $\mathrm{Zu}$ rückweisung für die Betroffenen einzustufen. Fast zwei Drittel der Befragten $(N=84)$ erhielten nach dem Berichten der traumatischen Erlebnisse keinerlei Reaktion der Vertrauensperson. Dabei sind die Unterschiede hinsichtlich des Geschlechts signifikant, $\chi^{2}(1, N=84)=7.96, p=.005$, $\phi=.308$ : Während $74.2 \%$ der Männer berichten, dass infolge des SichAnvertrauens nichts weiter passiert ist, trifft dies nur bei $40.9 \%$ der Frauen zu. In $56.8 \%(N=88)$ der Fälle berichten die Betroffenen, mit ihren Sorgen und Belastungen ,abgewimmelt“ worden zu sein. Jede/r Fünfte bzw. $(N=86)$ äußerte, aufgrund des Berichts über die sexuelle Misshand- 
lung öffentliche Denunziationen und Angriffe erlebt zu haben. $16.5 \%$ $(N=85)$ der Betroffenen schilderten, dass ihnen unterstellt wurde, den/die Täter/in verführt zu haben. Auch im Rahmen der offenen Antworten finden sich negative Reaktionen. Es wurde unter anderem berichtet, dass die eigenen Eltern an der Aussage zweifelten oder die Schuldfrage umgekehrt wurde: „Ich wurde gefragt, warum ich mich nicht gewehrt habe“. Auch die besondere gesellschaftliche Stellung der/des Täters/in bereitete den Betroffenen teilweise Schwierigkeiten, Unterstützung und Zuspruch zu finden. So berichtet eine Person, dass auf die Offenlegung ihrer sexuellen Misshandlung die Aussage: „Alles ist Lüge. Ein Priester macht so was nicht" folgte.

Die hohe Nennungshäufigkeit sowohl von unterstützenden als auch von negativen Reaktionen mutet zunächst widersprüchlich an. Es muss hierbei jedoch beachtet werden, dass die Reaktionen nicht nach dem/der jeweiligen Gesprächspartner/in einzeln, sondern nach allen erlebten Verhaltensweisen abgefragt wurden. Mehrfachantworten waren dabei möglich. Da der Missbrauch im Mittel 45 Jahre zurück liegt, ist anzunehmen, dass sich die Betroffenen im Laufe ihres Lebens verschiedenen Menschen anvertrauten und unterschiedliche Reaktionen erfuhren.

\section{III.2 Reaktionen der katholischen Kirche}

In den Medien, aber auch in einem aktuellen Bericht der UNO (UN, 2014) wurde immer wieder die Reaktion der katholischen Kirche auf das Bekanntwerden von Fällen sexuellen Missbrauchs kritisiert. Die Kirche habe das Ausmaß des Missbrauchs nie anerkannt und sich in den Reaktionen, zum Beispiel hinsichtlich rechtlicher Schritte, zurückgehalten (UN, 2014). Das Wegschauen und Verdrängen des Problems von institutioneller Seite soll mit dazu beigetragen haben, dass die Taten in einem solchen Umfang möglich waren. Aber auch innerhalb des Bewältigungsprozesses trägt die katholische Kirche Verantwortung. Benkert und Doyle (2009) weisen darauf hin, dass negative Äußerungen seitens der Kirche die psychischen Folgen der Betroffenen verschlimmern. Eine Bewältigung mittels des religiösen Glaubens, in dem religiös sozialisierte Betroffene in anderen Situationen Trost und Halt finden, wird dadurch erschwert. Deshalb war die Erfahrung der Betroffenen hinsichtlich des Umgangs der katholischen Kirche mit den Fällen des sexuellen Missbrauchs in der KFN-Untersuchung von besonderem Interesse. 
Um zu erfragen, wie das Verhalten der katholischen Kirche auf die Bekanntgabe der Missbrauchstat war, wurden den Befragten verschiedene mögliche Reaktionen zur Beantwortung vorgelegt. Die Betroffenen konnten angeben, inwiefern diese auf das, was sie erlebten, teilweise, völlig oder nicht zutrifft. In Abbildung 1 sind zunächst die positiven, das heißt unterstützenden oder hilfreichen Reaktionen mit ihren Nennungshäufigkeiten aufgeführt. ${ }^{10}$

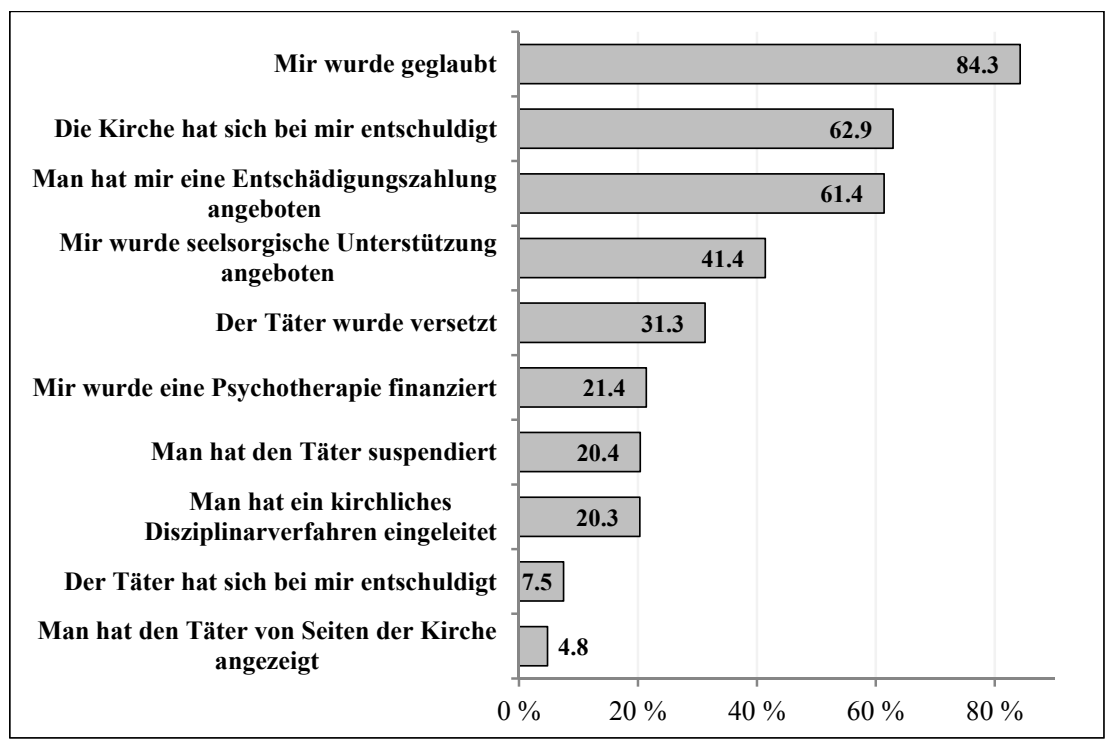

Abbildung 1. Positive Folgen der Mitteilung an die katholische Kirche $(N \geq 63$, bezogen auf jene Befragte, die sich an die Kirche gewandt haben)

Den meisten Betroffenen $(84.3 \%, N=70)$, die sich an die katholische Kirche wandten, wurde Glauben geschenkt und es erfolgte zumindest teilweise eine Entschuldigung $(62.9 \%, N=70)$. In den seltensten Fällen $(7.5 \%, N=66)$ hat sich jedoch die/der Täter/in selbst entschuldigt.

Ein Unterstützungsangebot in Form von Seelsorge erhielten $20.0 \%$ $(N=70)$ derjenigen, die den Kontakt zur Kirche suchten teilweise und 21.4 \% ganz. Größer ist dagegen die Zahl derer, die eine Entschädigungs-

10 Es zeigen sich keine signifikanten Unterschiede der Reaktionen der Kirche nach dem Geschlecht der Betroffenen. 
zahlung erhielten. $61.4 \%(N=70)$ der Befragten, die sich an die Kirche gewandt haben, bekamen eine Entschädigungszahlung. Mit $61.7 \%$ $(N=44)$ Nennungshäufigkeit erhielten die meisten Personen $5000 €$. Weitere $15.9 \%$ erhielten eine finanzielle Entschädigung in Höhe von $800 €$ bis $4000 €$. Zudem wurden in Einzelfällen auch deutlich höhere Summen als Entschädigung gezahlt. So geben $20.4 \%$ an, dass ihnen zwischen $7000 €$ und $20000 €$ ausgezahlt wurden. Es bleibt festzuhalten, dass $74.4 \%(N=43)$ derjenigen, die eine Entschädigung erhielten, mit der Höhe gar nicht und $14 \%$ eher nicht zufrieden waren. Lediglich fünf Personen waren eher bzw. voll zufrieden. Für $14.3 \%(N=70)$ der Befragten, die sich an die Kirche wandten, wurde eine Psychotherapie finanziert, wobei $7.1 \%$ teilweise eine Finanzierung erhielten (insgesamt $21.4 \%$ ).

Des Weiteren ist im Rahmen der Aufklärung der Reaktionen der katholischen Kirche von Interesse, wie diese mit der/dem Täter/in nach Bekanntwerden der Vorwürfe umging. In $20.3 \%(N=64)$ der berichteten Fälle wurde ein Disziplinarverfahren gegen die/den Täter/in eingeleitet. Häufiger jedoch (31.3\%) wurde die Option der Versetzung gewählt. Das Bekanntmachen des sexuellen Missbrauchs führte nur in $20.4 \%$ der Fälle zu einer Suspension der/des Täters/in. Drei Betroffene (4.8 \%) berichten, dass die Kirche mit einer Anzeige bei der Polizei reagierte.

Die Befragten berichten jedoch nicht nur Zurückhaltung bei der Ausführung von Konsequenzen für die/den Täter/in, sondern auch dezidiert negative und belastende Reaktionen seitens der Kirche (siehe Abbildung $2)$. Die Hälfte der Betroffenen $(N=65)$, die sich an die Kirche wandten, gibt an, dass ihre Aussage folgenlos blieb. Zudem fühlen sich $41.8 \%$ $(N=67)$ von den kirchlichen Vertretern/innen abgewimmelt. Schwerwiegend für die Betroffenen dürfte wohl auch eine Beweis- und Schuldumkehr als Reaktion auf die Meldung des Missbrauchs gewesen sein. 37.1\% $(N=70)$ der Betroffenen, die ihr Schweigen gegenüber der Kirche brachen, wurden mit der Anmerkung, keine Beweise für ihre Anschuldigungen zu haben, abgewiesen; in $7.1 \%(N=70)$ der Fälle wurde den Betroffenen eine (Mit-)Schuld vorgeworfen, indem unterstellt wurde, die/den Täter/in verführt zu haben oder verführen zu wollen. Ein Viertel $(26.8 \%, N=71)$ wurde gebeten, über das Geschehene zu schweigen. Dass die Mitteilung des Erlebten nicht immer vertraulich behandelt wurde, zeigt der Anteil derer, die der Aussage zustimmten „Ich wurde von Dritten öffentlich denunziert oder angegriffen" $(15.9 \%, N=69)$. 


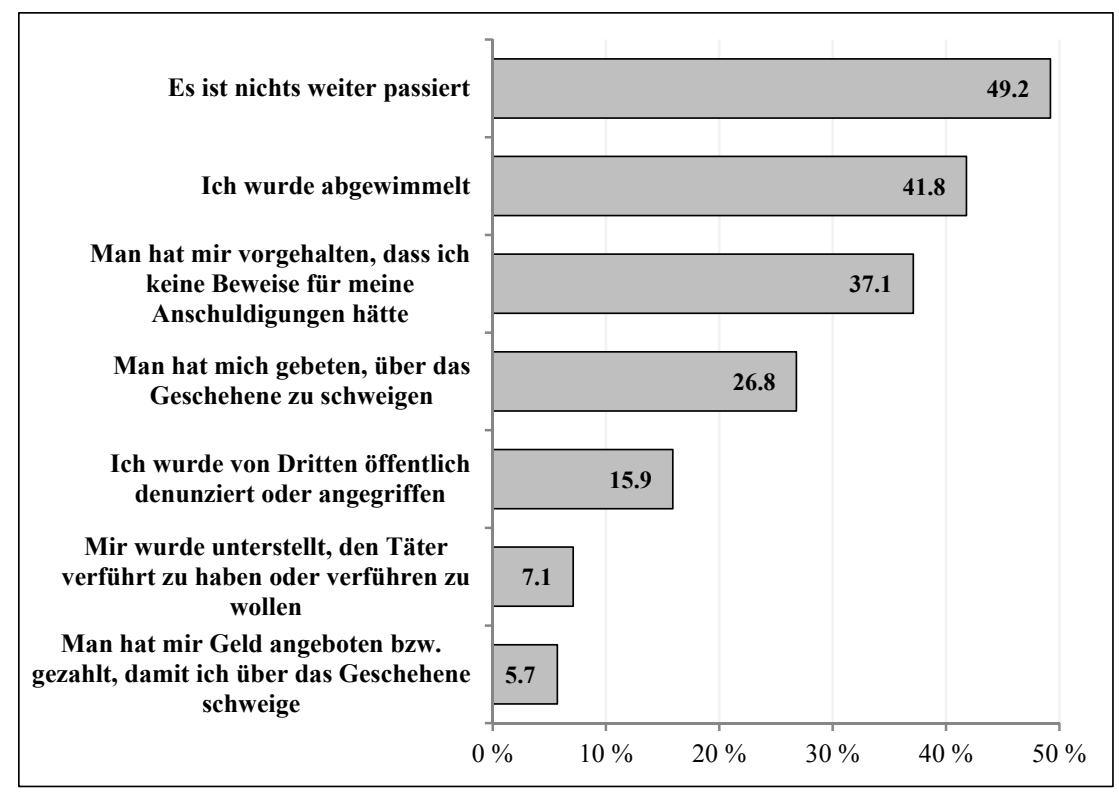

Abbildung 2. Negative Folgen der Mitteilung an die katholische Kirche ( $N \geq 63$, bezogen auf jene Befragte, die sich an die Kirche gewandt haben)

In dem offenen Antwortformat berichten einige Befragte ihre Enttäuschung bezüglich der kirchlichen Reaktionen. Diese äußerte sich lediglich knapp, ein wirkliches Gespräch, welches auf Verständnis und Bedauern beruhte, wurde vermisst. Ein/e Befragte/r meinte, einen deutlichen Unterschied in den Reaktionen der Kirche seit der medialen Debatte $2010 \mathrm{zu}$ spüren: Wurde 2002 ihre/seine Meldung noch skeptisch entgegengenommen, schenkte man ihr/ihm 2010 mehr Glauben.

Die Haltung der katholischen Kirche bezüglich der Missbrauchsfälle wird auch in der allgemeinen Zufriedenheit der Betroffenen mit dem Umgang der Kirche deutlich. So geben mehr als drei Viertel der Befragten an $(80.3 \%, N=71)$, dass sie gar nicht bzw. eher nicht zufrieden mit der Art und Weise sind, mit der sie nach Meldung des Missbrauchs von Seiten der katholischen Kirche behandelt wurden. Nur zwei Personen geben an, völlig zufrieden gewesen zu sein.

Insgesamt herrscht der Eindruck vor, dass die Kirche sich nicht ernsthaft mit den Vorfällen beschäftigt und sich nicht aufrichtig und kritisch mit den Missbrauchstaten in ihrer eigenen Institution auseinandersetzt. So berichten drei Viertel der Befragten $(76.1 \%, N=71)$ weiterhin, dass sie 
nicht den Eindruck hätten, die Kirche sei um Aufklärung bemüht. Zudem dominierte bei $64.8 \%(N=71)$ derjenigen, die sich an die Kirche wandten, das Gefühl, die Kirche wolle die Problematik lieber verdrängen und vor allem vertuschen. Allerdings besteht in Bezug auf diese Frage keine einheitliche Tendenz, denn für $35.2 \%$ trifft diese Aussage nicht bzw. eher nicht zu. Insgesamt hätten sich die meisten Betroffenen $(72.9 \%, N=70)$ mehr Unterstützung von der katholischen Kirche gewünscht.

Die häufige Nennung sowohl positiver als auch negativer Reaktionen könnte mit den unterschiedlichen kirchlichen Gesprächspartner/innen zusammenhängen. Die Befragten konnten angeben, mit welchen Personen sie von der katholischen Kirche gesprochen haben. Dabei waren Mehrfachantworten möglich. Es zeigt sich, dass $72.7 \%$ derjenigen, die sich (eventuell neben anderen Personen der Kirche) an eine/n Missbrauchsbeauftragte/n der Kirche wandten, der Aussage zustimmen, die Kirche habe sich bei ihm bzw. ihr entschuldigt, wohingegen dies nur $46.2 \%$ der Befragten bejahten, die mit anderen Personen der katholischen Kirche gesprochen haben. Dieser Unterschied ist signifikant, $\chi^{2}(1, N=70)=4.94$, $p=.044, \phi=.247$. Ebenso wurde Betroffenen, die sich an eine/n Missbrauchsbeauftragte/n der Kirche wandten, mehr seelsorgerische Unterstützung $(52.3 \%)$ angeboten als solchen, die mit anderen Menschen der katholischen Kirche sprachen $(23.1 \%)$. Auch dieser Unterschied ist signifikant, $\chi^{2}(1, N=70)=5.74, p=.017, \phi=.286$. Weiter unterscheiden sich die Befragten, die ein Gespräch mit einer/m Missbrauchsbeauftragten hatten, signifikant dadurch, dass nur $15.9 \%$ von ihnen angeben, von einer Person der Kirche gebeten worden $\mathrm{zu}$ sein, über das Geschehene $\mathrm{zu}$ schweigen, während $44.4 \%$ der Befragten dies berichten, die mit anderen Personen als einer $/ \mathrm{m}$ Missbrauchsbeauftragten der Kirche sprachen, $\chi^{2}(1, N=71)=6.95, p=.008, \phi=.313$. Dabei ist anzunehmen, dass nicht die Missbrauchsbeauftragten selbst zum Schweigen aufforderten, sondern es sich bei den genannten $15.9 \%$ eher um Befragte handelt, die neben dem/r Missbrauchsbeauftragten auch mit anderen Personen der katholischen Kirche gesprochen haben.

Bei der Interpretation der Befunde sollte berücksichtigt werden, dass die Institution eines/r Missbrauchsbeauftragen neu ist. Somit kann die Nennung positiver Reaktionen im Zusammenhang mit Gesprächen mit Missbrauchsbeauftragten einerseits ein Indiz dafür sein, dass Vertreter/innen der Kirche sich heute anders gegenüber den Betroffenen verhalten als früher. Andererseits könnten die Ergebnisse aber auch ein Hinweis dafür sein, dass es Unterschiede zwischen den Personen innerhalb der ka- 
tholischen Kirche gibt. Da die Befragten die Möglichkeit der Mehrfachantworten hatten, kann dies nicht abschließend geklärt werden.

Die Reaktionen der katholischen Kirche können jedoch nicht nur nach dem/der Ansprechpartner/in sondern auch nach dem Zeitpunkt der ersten Kontaktaufnahme mit der Kirche differenziert werden. Die Befragten konnten angeben, ob sie sich unmittelbar nach der ersten Missbrauchshandlung, während der Zeit des sexuellen Missbrauchs oder nach dem sexuellen Missbrauch bei der Kirche gemeldet haben. Diejenigen, die sich nach der Tat meldeten, wurden aufgefordert, das Jahr zu nennen. Bei denjenigen, die sich unmittelbar nach oder während des Missbrauchs meldeten, wurde das Jahr herangezogen, in welchem der Missbrauch begann. Danach unterschieden, ob sich im Zeitraum von 1949 bis 2009 oder ab 2010 an die Kirche gewandt wurde, zeigt sich, dass $75.6 \%$ derjenigen, die sich ab 2010 bei der Kirche meldeten, berichten, dass die Kirche sich entschuldigt habe, wohingegen dies nur $46.4 \%$ meinen, die sich davor an die Kirche wandten. Dieser Unterschied ist signifikant, $\chi^{2}(1, N=69)=6.13$, $p=.013, \phi=.298$. Auch das Angebot von seelsorgerischer Unterstützung bejahen mit $53.7 \%$ eher diejenigen, die ab 2010 die Kirche ansprachen, wohingegen nur $25.0 \%$ derjenigen, die vor 2010 Kontakt aufnahmen dies erhielten, $\chi^{2}(1, N=69)=5.61, p=.018, \phi=.285$. Auf der anderen Seite zeigen sich auch negative Konsequenzen eher vor 2010. Die Aufforderung über das Geschehene zu schweigen, erlebten $12.2 \%$ der Befragten, die sich ab 2010 an die Kirche wandten, jedoch $48.3 \%$ der Betroffenen, die schon davor Vertreter/innen der Kirche ansprachen, $\chi^{2}(1, N=70)=11.18$, $p=.001, \phi=.400$. Öffentliche Denunziationen als Folge der Meldung bei der Kirche erlebten $7.5 \%$ der Befragten ab 2010, $28.6 \%$ dagegen, die vor 2010 mit der Kirche sprachen, $\chi^{2}(1, N=68)=5.39, p=.020, \phi=.282$. Wenn auch nicht die Reaktionen direkt zu dem Zeitpunkt verbunden erhoben wurden, so sind diese Ergebnisse dennoch ein Hinweis darauf, dass es einen Wandel in der Haltung der Kirche gegenüber den Betroffenen gibt. Dies könnte eine Erklärung dafür sein, dass insgesamt sowohl positive als auch negative Folgen genannt werden und heute $68.0 \%(N=100)$ der Betroffenen angeben, anderen zu raten, sich mit dem Erlebten an die katholische Kirche zu wenden.

\section{III.3 Reaktionen der Polizei}

Die Betroffenen wurden explizit nach dem Verhalten der Polizei gefragt, sofern sie sich an diese wandten. Von den Befragten, die Kontakt zur Po- 
lizei aufnahmen, stimmen $90.3 \%(N=31)$ teilweise oder völlig $z u$, dass ihnen geglaubt wurde (siehe Abbildung 3). $28.6 \%(N=32)$ geben an, dass ihnen aufgrund der Anzeige seelsorgerische Unterstützung angeboten wurde. Nur eine Person berichtet, dass sich die/der Täter/in aufgrund der Anzeige entschuldigte. Allerdings führte in den meisten Fällen die Anzeige zu keinem für die Betroffenen nennenswerten Ergebnis, vielmehr hatten sie das Gefühl, dass nichts dabei heraus kam $(79.3 \%, N=29)$ bzw. nichts weiter passierte $(77.4 \%, N=31)$. Obwohl fast alle Anzeigenden meinen, ihnen wurde Glauben geschenkt, berichten dennoch $38.7 \%$ $(N=31)$ abgewimmelt worden zu sein. In $34.4 \%(N=32)$ der Fälle, die der Polizei bekannt wurden, wurde den Betroffenen mitgeteilt, dass das Fehlen von Beweisen problematisch sei; in weiteren $15.1 \%(N=33)$ der Fälle, wurden die Betroffenen von der Polizei zum Schweigen über das Geschehene aufgefordert. Einer Person $(N=33)$ wurde eine Mitschuld im Gespräch bei der Polizei gegeben, indem ihr vorgeworfen wurde, die/den Täter/in selbst verführt zu haben. Die Anzeige bei der Polizei führte bei sieben Befragten $(21.9 \%, N=32)$ zu negativen Reaktionen Dritter, welche aus öffentlichen Denunziationen bestanden.

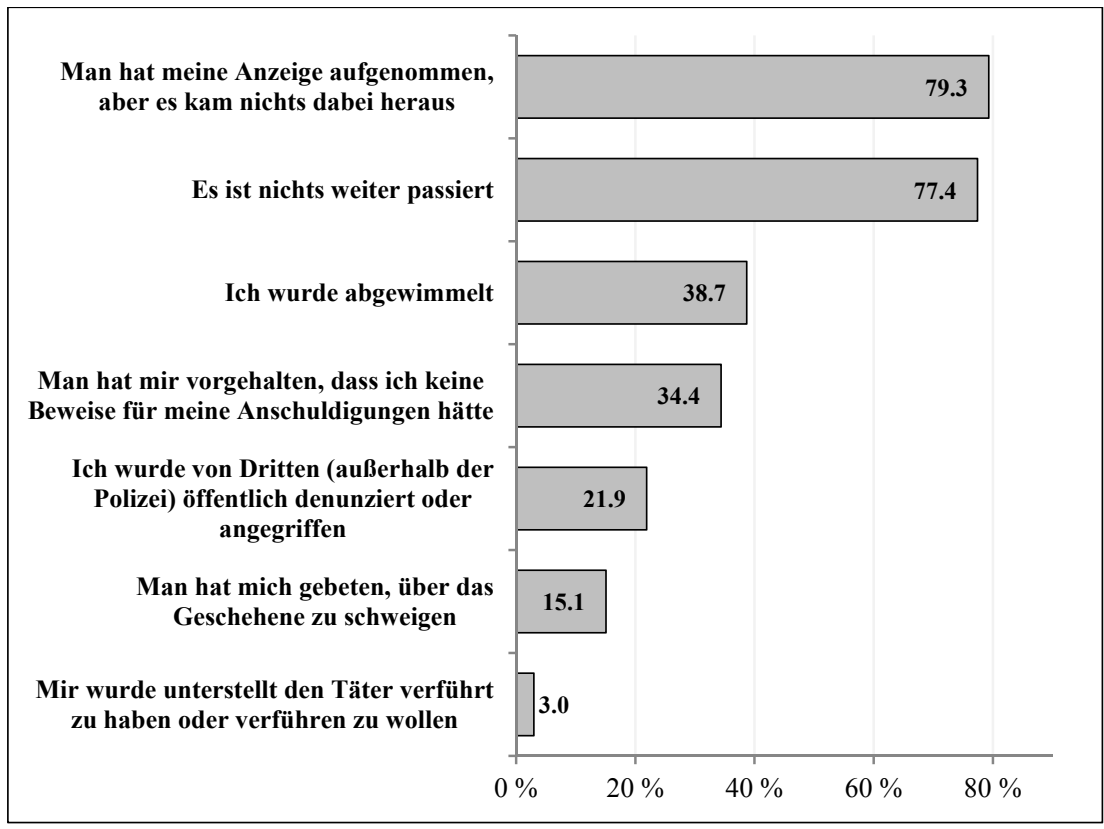

Abbildung 3. Negative Folgen der Strafanzeige $(N=29$, bezogen auf jene Befragte, deren Fälle der Polizei bekannt wurden; Angaben in \%) 
Auch in dem offenen Antwortformat häuften sich Enttäuschungen bezüglich der Folgen einer Anzeige. So berichtet eine Person, aufgrund ihres Viktimisierungsalters von 12 Jahren von der Polizei nicht ernst genommen, sondern als ,passiver Beteiligter" eingestuft worden zu sein. Weiter beklagt ein/e Betroffene/r, dass er bzw. sie sich von einer Anzeige vor allem erhoffte, weitere Taten verhindern zu können, da die/der Täter/in noch im Dienst war. Dennoch führte die Anzeige nur zu einer Einstellung des Verfahrens aufgrund der Verjährung der Straftat.

Das Problem der Verjährung der Straftat wurde häufig geschildert. Durch das Vorherrschen zeitlich sehr verzögerter Anzeigen wurde das Verfahren in $84.4 \%(N=32)$ der angezeigten Fälle wegen der Verjährung der Straftat eingestellt. Nur bei acht Anzeigen $(N=32)$ kam es zu einer Anklage der/s Täters/in. Dabei dauert das Verfahren in drei Fällen $(N=29)$ noch an, zwei Täter/innen $(N=29)$ wurden zu einer Freiheitsstrafe mit Bewährung und ein/e Täter/in $(N=29)$ zu einer Freiheitsstrafe ohne Bewährung verurteilt. Eine weitere Person $(N=28)$ erhielt eine Geldstrafe, in einem anderen Fall $(N=27)$ kam es zu einem Freispruch.

Es bleibt die Frage zu klären, warum sich so wenige Betroffene dieser schwerwiegenden Straftat an die Polizei wandten. Es scheint vor allem zwei verschiedene Hemmfaktoren zu geben: Zum einen könnte eine Anzeige ergebnislos erscheinen (siehe Abbildung 4), das heißt keinen Vorteil mit sich bringen. Diese Gründe sollen zuerst dargestellt werden. Zum anderen könnte die Anzeige und das daraus resultierende juristische Verfahren Angst bereiten (siehe Abbildung 5), was in einem nächsten Schritt erläutert wird. Daran anschließend werden noch weitere Gründe für eine Nichtanzeige genannt (siehe Abbildung 6).

Für die Mehrheit der Betroffenen schien eine Anzeige bei der Polizei ergebnislos (siehe Abbildung 4). So geben die Befragten an, dass sie das Erlebte eher für sich geklärt haben $(39.3 \%, N=61)$ und es ihnen nichts bringen würde, es bei der Polizei zu melden (43.3\%,N=63). Die Gründe dafür, dass ihnen eine Anzeige nicht hilft, sehen die Betroffenen darin, dass ihnen niemand glauben $(45.1 \%, N=62)$ bzw. es niemand verstehen $(37.1 \%, N=65)$ würde. Die meisten $(55.4 \%, N=65)$ empfinden die Tatsache, nichts beweisen zu können, als Hindernis für die Kontaktaufnahme mit der Polizei.

Zudem spielt der Fakt, dass die/der Täter/in zur Kirche gehört, für ein Viertel der Befragten $(N=66)$ auch hier eine Rolle für das zurückhaltende Anzeigeverhalten. Fast ebenso häufig wird angegeben $(22.7 \%, N=66)$, dass man die Tat am liebsten auf sich beruhen lassen möchte. 


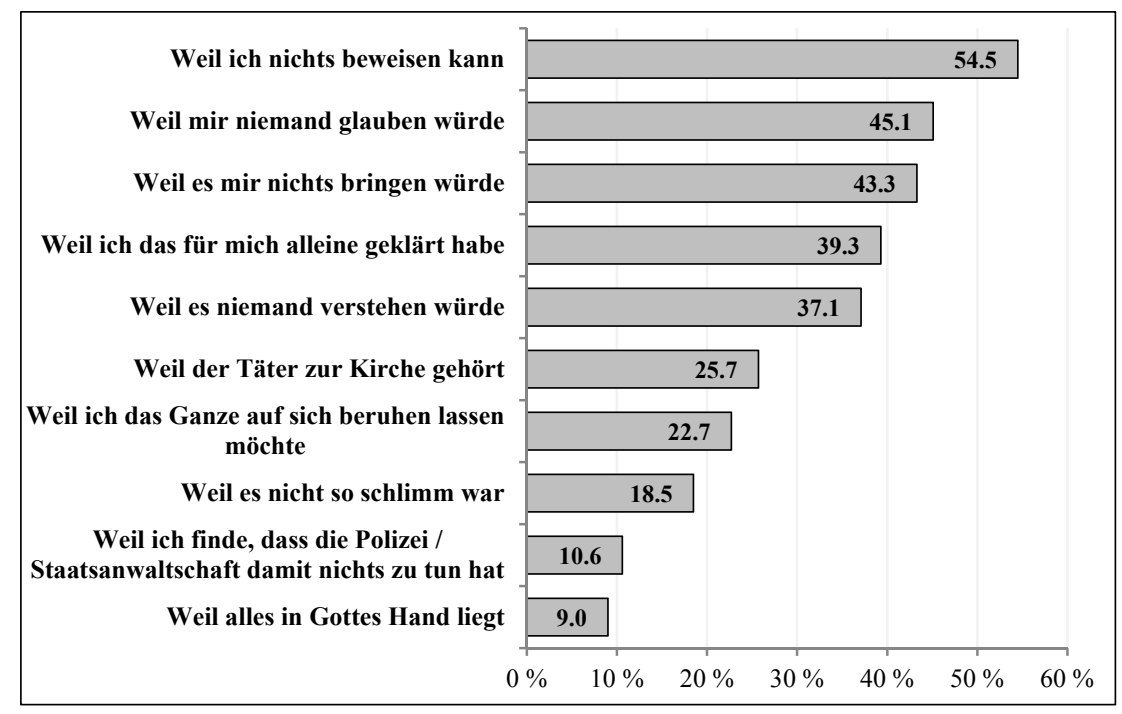

Abbildung 4. Gründe für eine Nichtanzeige ( $N \geq 61$, bezogen auf jene Befragte, deren Fälle der Polizei nicht bekannt wurden; Angaben in \%)

Seltener $(18.5 \%, N=65)$ äußern die Betroffenen das Empfinden, dass das Ausmaß der Tathandlungen nicht schlimm genug für eine Anzeige gewesen sei. Das Infragestellen der Zuständigkeit der Polizei $(10.6 \%, N=66)$, teilweise auch durch ein Zusprechen der göttlichen Verantwortung (9\%, $N=66$ ), liegt nur bei sehr wenigen Befragten als Grund dafür vor, von einer Strafanzeige abgesehen zu haben

Die Befragten berichten zudem verstärkt, dass sie durch verschiedene Ängste von einer Strafanzeige abgehalten wurden (siehe Abbildung 5). Als vorherrschend werden die Angst vor dem Verfahren $(42.4 \%, N=66)$ und vor der Öffentlichkeit $(33.4 \%, N=66)$ kategorisiert. Nahezu ein Drittel der Befragten $(32.3 \%, N=65)$ gibt an, aus Rücksicht auf die eigene Familie ein gerichtliches Verfahren zu meiden. Auch die Drohung der/s Täters/in hielt einige Befragte $(18.2 \%, N=66)$ von rechtlichen Schritten ab. Die Sorge um die/den Täter/in hingegen stellt in der Regel kein Hindernis dar $(7.7 \%, N=65)$. 


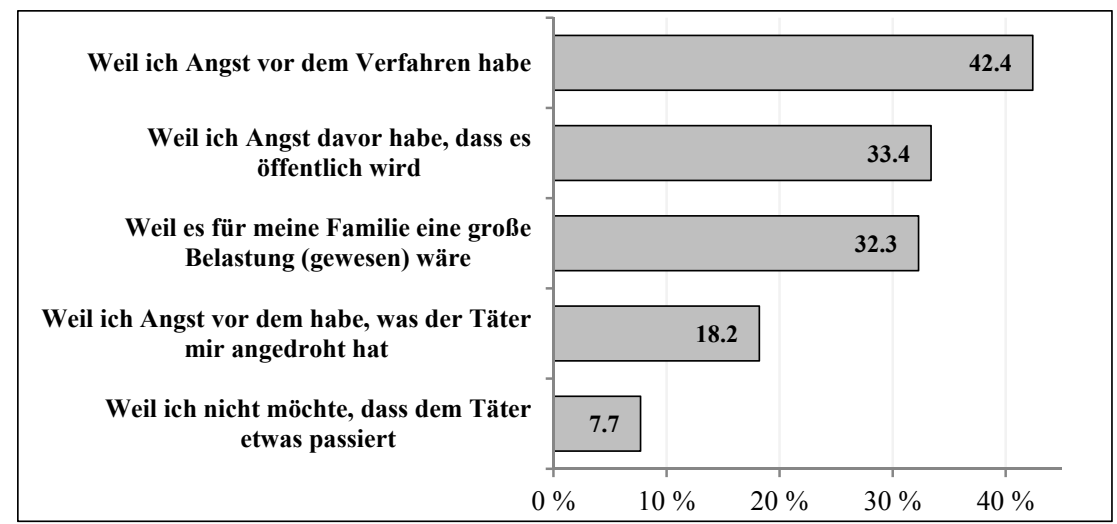

Abbildung 5. Gründe für eine Nichtanzeige $(N \geq 65$, bezogen auf jene Befragte, deren Fälle der Polizei nicht bekannt wurden; Angaben in \%)

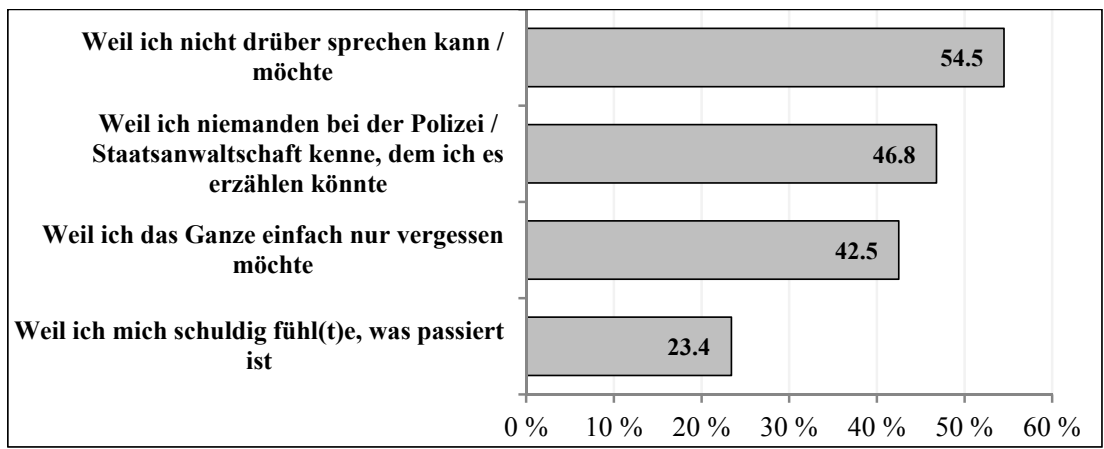

Abbildung 6. Weitere Gründe für eine Nichtanzeige $(N \geq 62$, bezogen auf jene Befragte, deren Fälle der Polizei nicht bekannt wurden; Angaben in \%)

Der Strafanzeige bei der Polizei steht jedoch bei vielen Betroffenen auch entgegen, dass sie sich selbst nicht in der Lage sehen, eine solche zu erstatten (siehe Abbildung 6). Bei 46.8\% $(N=62)$ der Befragten fehlt eine Vertrauensperson bei der Polizei, um die traumatischen Erlebnisse zu berichten. Die Meisten $(54.5 \%, N=66)$ fühlen sich nicht fähig, überhaupt über das Erlebte zu sprechen. Bei fast einem Viertel der Betroffenen $(23.4 \%, N=64)$ spielen auch eigene Schuldgefühle eine Rolle für die Hemmung, eine Anzeige zu erstatten. Oft wiegt der Wunsch, die gesamten missbrauchsbezogenen Erinnerungen zu vergessen, stärker als der Wunsch nach justizieller Gerechtigkeit $(42.5 \%, N=66)$. 
Festgehalten werden kann, dass nur sehr wenige Befragte die Polizei aufsuchten. Die Bedenken, dass dieser Schritt nichts bringen würde, die Angst vor den Folgen und die Schwierigkeit, überhaupt über den sexuellen Missbrauch zu sprechen, stellten für die Meisten ein Hindernis dar. Dennoch würden $86.5 \%$ der Befragten $(N=83)$ anderen Betroffenen raten, sich an die Polizei zu wenden. Dies deutet darauf hin, dass der Wunsch nach juristischen Konsequenzen durchaus vorhanden ist.

\section{Exkurs: Rechtsansprüche nach dem Opferentschädigungsgesetz}

Neben einer polizeilichen Anzeige besteht für die Betroffenen sexuellen Missbrauchs auch die Möglichkeit eines Antrags auf eine staatliche Entschädigungszahlung nach dem Opferentschädigungsgesetz (OEG). ${ }^{11}$ Fast die Hälfte der Befragten (47.8\%) gibt an, nicht im Laufe ihres Lebens über die Möglichkeit einer Entschädigung nach dem OEG informiert worden zu sein (Hellmann \& Bartsch, 2014). In $29.8 \%(N=94)$ der Fälle wurde ein Antrag auf eine Entschädigung nach dem OEG gestellt, wobei $24.5 \%$ der Befragten den Antrag selbst gestellt haben und bei $5.3 \%$ dieser von einer anderen Person bzw. Organisation eingereicht wurde (Hellmann \& Bartsch, 2014).

Die Betroffenen, bei denen ein OEG-Antrag entweder von ihnen selbst oder einer anderen Person gestellt wurde, konnten angeben, inwiefern sie vorgelegten Aussagen zu dem Verfahren zustimmen (Hellmann \& Bartsch, 2014). Dabei meinen $64.3 \%$, dass sie eher nicht bzw. gar nicht fair behandelt wurden. $50.0 \%$ halten den Ausgang des Verfahrens gar nicht bzw. eher nicht gerecht. Die meisten Betroffenen (60.7\%), die ein OEGVerfahren erlebten, geben an, sich gar nicht bzw. eher nicht informiert zu fühlen. Dies scheint nicht zuletzt auch an der Betreuung des/der zuständigen Sachbearbeiters/in zu liegen. 46.4\% lehnen die Aussage ab, von dem/der Sachbearbeiter/in gut betreut worden zu sein. Zwar würden $64.3 \%$ anderen Betroffenen raten, einen Antrag nach dem OEG zu stellen, dennoch sehen die meisten Befragten (75.0 \%) einen Verbesserungsbedarf bei dem Verfahren. Vor allem wurde das Verfahren von $57.1 \%$ als sehr langwierig beschrieben.

11 Eine ausführliche Auswertung der Ergebnisse zum OEG findet sich bei Hellmann und Bartsch (2014). 
In dem offenen Antwortformat beschreiben die Betroffenen, das Verfahren als retraumatisierend, entwürdigend und verletzend erlebt zu haben: „Die Schilderung der Umstände sind jedes Mal eine Tortour. Ebenso die immer wiederkehrenden Gutachten. Alles kommt wieder hoch, wird aktualisiert. Ich brauche Monate um mich davon zu erholen. Wenn ich das Geld nicht dringend benötigen würde, würde ich liebend gern darauf verzichten." Ein/e andere/r Befragte gibt an, aufgrund der Retraumatisierungen den Antrag zurückgezogen zu haben. Auch die lange Verfahrensdauer wird betont. So dauert in einem Fall das Verfahren seit sechs Jahren an. Abgesehen von der psychologischen Belastung, schildern einige Befragte auch andere Schwierigkeiten bei der Antragstellung. So sei das Verfahren undurchsichtig und die Begründungen und Entscheidungen teilweise nicht nachvollziehbar. Ein/e Befragte/r berichtet, dass nach Antragstellung lange Zeit nichts geschah und erst nach seiner/ihrer wiederholten Eigeninitiative etwas passierte: „Ein langer Irrweg bis ich an die Richtigen gekommen bin“.

Zum Zeitpunkt der Befragung waren $25.0 \%(N=28)$ der Anträge bewilligt, wohingegen $14.3 \%$ abgelehnt wurden (Hellmann \& Bartsch, 2014). In insgesamt $25.0 \%$ der Fälle dauert das Verfahren noch an, da es nach einer ersten Bewilligung oder Ablehnung des Antrags zu einem Widerruf kam (Hellmann \& Bartsch, 2014). Sechs Betroffene (21.4\%) warten noch auf die Entscheidung in erster Instanz (Hellmann \& Bartsch, 2014). Dies bedeutet letztlich, dass von 104 Betroffenen des sexuellen Missbrauchs durch katholische Geistliche nur sieben von einem bewilligten OEG-Antrag berichten; dies sind $6.7 \%$.

\section{Fazit}

Das vorliegende Kapitel stellte neben dem Offenbarungsverhalten und den Einflussfaktoren der Offenbarungsbereitschaft die erlebten Reaktionen von Vertrauenspersonen, Kirche und Polizei auf die Meldung des sexuellen Missbrauchs dar. Dabei zeigte sich, dass es nur sehr wenigen der Befragten als Kind gelang, den „cocoon of fear“ (Benkert \& Doyle, 2009, S. 237) zu brechen und mit einer anderen Person über die Tat zu sprechen. Die Hälfte der Befragten sprach erst mehr als zehn Jahre nach dem Missbrauch das erste Mal darüber. Dementsprechend erhielten die Betroffenen, wenn überhaupt, erst spät professionelle Unterstützung, zum Beispiel in Form von Psychotherapien. Multiple Faktoren beeinflussen, ob ein Kind über ein solches Erlebnis spricht oder nicht. Die Unterschiede in dem Of- 
fenbarungsverhalten je nachdem, ob ein Kind eine zugewandte Erziehung erlebte, sind jedoch Hinweise dafür, dass das soziale Umfeld des Kindes eine wichtige Rolle hierbei spielt. Kinder brauchen Ermutigung dazu, das Erlebte zu hinterfragen und sich Hilfe zu suchen. Dazu ist es notwendig, dass sie Menschen kennen, denen sie vertrauen und sich somit auch anvertrauen können. Das Hinzuziehen des KFN-Viktimsurvey von 1992 hat jedoch gezeigt, dass sich die Betroffenen sexuellen Missbrauchs von anderen Personen ihres Alters stark in Hinblick auf die Sozialisation durch die Eltern unterscheiden. So erlebten Letztgenannte viel mehr Zuwendung und um einiges weniger gewaltvolle Erziehungsstile. Inwiefern diese Unterschiede im Aufwachsen sich schon auf die Wahl des Betroffenen von dem/der Täter/in auswirkte, kann hier nicht geklärt werden. Es kann jedoch festgehalten werden, dass das Umfeld der Betroffenen nach dem sexuellen Missbrauch kaum eine Unterstützung darstellte; in den meisten Fällen gab es für das Kind noch nicht einmal eine Person, der er bzw. sie sich überhaupt anvertraute.

Es sind jedoch nicht nur allein die primären Erziehungspersonen, denen Verantwortung im weiteren Viktimisierungsprozess zukommt. Da der sexuelle Missbrauch von katholischen Geistlichen verübt wurde, kann das Erlebte nicht losgelöst von der institutionellen Einbettung der Kirche betrachtet werden. So wird deutlich, dass es den Betroffenen vor allem auch in Orten starker katholischer Prägung schwer fiel, sich jemanden anzuvertrauen. Des Weiteren wird ebenso in offenen Antwortformaten immer wieder auf die erhöhte Stellung der/des Täters/in durch die religiöse Funktion, die diese/r innehatte, hingewiesen. Im Laufe ihres Lebens wandten sich die Betroffenen häufig an die Kirche und berichteten von dem Erlebten. Der Kirche wurden sogar mehr Fälle mitgeteilt, als der Polizei. Dies ist ein Hinweis darauf, dass die Befragten ein Eingreifen und Handeln der Kirche erwarten.

Die Reaktionen, die die Befragten, die sich an die Kirche wandten, erfuhren, scheinen jedoch nicht immer befriedigend gewesen zu sein. Betroffenen wurde Mitschuld gegeben, sie wurden zum Schweigen über das Erlebte aufgefordert und die Konsequenzen für die/den Täter/in waren meist gering. Erst in den letzten Jahren scheint sich die Haltung der Kirche zu wandeln. So zeigen die Daten, dass die Personen, die mit Missbrauchsbeauftragten der Kirche bzw. ab 2010 zu der Kirche Kontakt aufnahmen, deutliche positivere Reaktionen wie Verständnis und Entschuldigungen erhalten haben.

Die allgemeinen Reaktionen infolge der Meldung des Missbrauchs waren ebenso sehr unterschiedlich. So geben die Befragten an, dass ihnen 
mehrheitlich geglaubt wurde und sie emotionale Unterstützung erfuhren. Jedoch erlebte auch über die Hälfte der Befragten das Gefühl ,abgewimmelt" worden zu sein und musste das Ausbleiben weiterer Schritte oder Konsequenzen registrieren. Es kann vermutet werden, dass die Unterschiedlichkeit der Reaktionen des Umfelds mit den gesellschaftlichen Veränderungen einhergeht und sich auch hier ein Wandel mit der Zeit vollzog.

Es scheint, dass die Hindernisse, im sozialen Nahraum die Tat zu berichten, sich auch auf die Möglichkeit der Strafverfolgung auswirkten und eine Erklärung für die geringe Anzeigequote darstellen. Wie oben dargelegt, wurde nur ein sexueller Missbrauch zeitnah zur Tatzeit angezeigt. Viele strafrechtliche Ermittlungen scheiterten an der Verjährungsfrist. Dies dürfte sicherlich ein Grund dafür sein, dass Befragte sich enttäuscht über den Ausgang der Anzeige zeigen, auch wenn viele meinen, dass ihnen von Seiten der Polizei geglaubt wurde.

\section{Literatur}

Benkert, M. \& Doyle, T. P. (2009). Clericalism, religious duress and its psychological impact on victims of clergy sexual abuse. Pastoral Psychology, 58, 223-238.

Briere, J. N. \& Elliott, D. M. (1994). Immediate and long-term impacts of child sexual abuse. The Future of Children, 4, 54-69.

Deutsche Bischofskonferenz (2013). Bericht zum Abschluss der Tätigkeit der Hotline der Deutschen Bischofskonferenz für Opfer sexuellen Missbrauchs Teil 2. Verfügbar unter http://www.dbk.de/fileadmin/redaktion/diverse_downloads/presse_ 2012/2013-008e-Taetigkeitsbericht-Hotline_Teil-2.pdf [15.01.2014]

Elliott, A. N. \& Carnes, C. N. (2001). Reactions of nonoffending parents to the sexual abuse of their child: A review of the literature. Child Maltreatment, 6, 314-331.

Hellmann, D. F. \& Bartsch, T. (2014). Berücksichtigung der Belange von Betroffenen sexuellen Missbrauchs im Opferentschädigungsgesetz: Gleiches Recht für alle? Monatsschrift für Kriminologie und Strafrechtsreform, 97, 131-149.

Lueger-Schuster, B. (2012). Psychotraumatologische Fragestellungen zu Gewalt und Missbrauch in der Katholischen Kirche. Wien: Universität Wien.

UN - Committee on the Right of the Child. (2014). Concluding observations on the second periodic report of the Holy See. Verfügbar unter: http://tbinternet.ohchr.org/ _layouts/treatybodyexternal/Download.aspx?symbolno $=\mathrm{CRC} \% 2 \mathrm{fC} \% 2 \mathrm{fDEU} \% 2 \mathrm{fC}$ O\%2f3-4\&Lang=en [04.02.2014]

Wetzels, P. (1997). Gewalterfahrungen in der Kindheit: Sexueller Mißbrauch, körperliche Mißhandlung und deren langfristige Konsequenzen. Baden-Baden: Nomos.

Wetzels, P. \& Brettfeld, K. (2003). Auge um Auge, Zahn um Zahn? Migration, Religion und Gewalt junger Menschen. Münster: LIT-Verlag. 
